\title{
Insight in spastic musculoskeletal structures in cerebral palsy: impaired or compensatory structural changes?
}

\author{
Luigi Di Lorenzo1,2 \\ Alfonso Maria Forte 2 \\ Francesco Forte ${ }^{2}$
}

1 Rehabilitation Unit, Movement Disorders Treatment
C., Neuroscience Department, "RUMMO" Hospital,
Benevento, Italy
2 Biomedical Research Centre, Gruppo Forte Salerno,
Italy

Dear Editor

we read with great interest the recently published paper by Gagliano et al. ${ }^{1}$ which raises several interesting points for discussion and we would like to commend the authors for mainly "thinking outside the box of a basic researcher". The authors studied the effect of spasticity on tendons from the gracilis and semitendinosus muscles from cerebral palsy (CP) and healthy subjects, observing an increased cellularity, cell rounding, and hypervascularity and lipoid degeneration in tendon samples from CP patients. They report evidencies of an increased collagen content and an increased glycosaminoglycan content in CP tendons, concluding that these results were a consequence of spasticity, which may be considered a chronic, persisting and repetitive loading of tendons, inducing extra cellular matrix (ECM) remodelling and an adaptive response to increased functional demands. In a recent paper, de Bruin summarized the evidence about movement limitations of spastic patients and underlying how a multidimensional problem requires a multidimensional and a multidisciplinary approach. He suggests to use microscopic and macroscopic perspectives and to work together with experts from different specialities ${ }^{2}$. Three main structures define the mechanics of the musculoskeletal system: muscle, connective tissue and bone ${ }^{2}$. In vivo, all these structures interact with each other, adapting structure to mechanics and viceversa. Acute exercise can lead to increased collagen synthesis in both tendon and muscle, to an adaptive response in the connective tissue of the muscle-tendon unit, and to increased expression of collagen-inducing growth factors in tendon and muscle. This is in line with the hypothesis that mechanical loading of these tissues induces collagen expression via an increased expression of certain growth factors ${ }^{2,3}$. Importantly, the tendon tissue response, contrary to muscle, is reported to be independent of stress levels/contraction type, indicating that this tissue is less sensitive than skeletal muscle to differences in mechanical stim$\mathrm{uli}^{3}$. The collagen fibre network is a major contributor to the coherence and tensile strength of normal skeletal muscle: immobilization results in marked deterioration of function and biomechanical properties of the immobilized skeletal muscle ${ }^{4}$. "Cerebral Palsy" is an umbrella term covering a group of non-progressive, but often adapting, motor impairments secondary to different lesions. CP patients adapt their movement strategy to execute certain challenging tasks ${ }^{5}$, developing differences in motor pattern between children with diplegia and those with hemiplegia that could, for example, consistent with a better overall selectivity in lower limb in unilateral $\mathrm{CP}{ }^{6}$. The maintenance of range of motion seems to be essential because blood flow to the skeletal muscle is tightly regulated by its metabolic demands through control of the nitric oxide receptor causing local vasodilation ${ }^{7}$. While recent findings indicate also that exercises induce adaptive response in tendon and muscle connective tissue, involving increased synthesis of collagen ${ }^{7}$, others suggest that the tendon tissue response, contrary to muscle, is independent of stress levels/contraction type, indicating that this tissue is less sensitive than skeletal muscle to differences in mechanical stimulus ${ }^{8}$. In this contest ${ }^{1}$, a casual reader might think to base the "core" of CP management on spasticity treatment if assuming that it directly influence the ECM composition. Indeed, these adaptations seem to be reversible and Langevin indicates that tissue contraction and relaxation may result in a dynamic, body-wide pattern of cellular activity ${ }^{9}$. A constrained movement pattern during the work task not only leads to a restricted load distribution between muscles but could also have deleterious effects on specific subsets of muscle fibers. Altered movement patterns of CP patients have already been suggested not to be purely pathological ${ }^{2,6}$ and understanding of these characteristics and how structure and mechanics interact can help understanding pathologies of movement. In conclusion, from a clinical point of view we hope that our letter should widen the lens rather than narrow the focus because we believe that spastic myotendineous pattern and impaired structural changes in spastic musculoskeletal structures should have to be studied together and possibly described as compensatory and partially reversible structural changes in an altered motor pattern. This because in compensation model, we expect muscle that are thought to be impaired to still have the ability to performance.

\section{References}

1. Gagliano N, Menon A, Martinelli C, et al. Tendon structure and extracellular matrix components are affected by spas- 
ticity in cerebral palsy patients. Muscle Ligament Tendon $\mathrm{J}$ 2013; 3(1):42-50.

2. de Bruin M. Connecting the dots: musculoskeletal adaptations in Cerebral Palsy. 2013 Pages 186 ISBN 9789090273488 AMC-UvA. Free retrieved online (last access 16 Sept 2013) at: http://dare.uva.nl/en/record/441513.

3. Heinemeier KM. Adaptation of tendon and muscle connective tissue to mechanical loading. Involvement of collageninducing growth factors. Institute of Sports Medicine Bispebjerg Hospital Copenhagen. Freeree retrieved on line (last access 10 July 2013) at http://www.ismc.dk/pages/_

4. Jarvine TA, Jozsa L, Kannus P, Jarvinen M, Jarvine TA. Organization and distribution of intramuscular connective tissue in normal and immobilized skeletal muscles. An immunohistochemical, polarization and scanning electronmicroscopic study. Muscle Res Cell Motil 2002; 23(3):245254.

5. Rosenbaum P, Paneth N, Leviton A, et al. A report: The definition and classification of cerebral palsy. Developmenta Medicine \& Child Neurology 49:8-14. Corrected in Rosenbaum P, Paneth N, Leviton A, Goldstein M, Bax M, Damiano D, Dan B, Jacobsson B. A report: The definition and classification of cerebral palsy. Developmental medicine and child neurology. Supplement 2007; 109:8-14.

6. Kreulen M. Myofascial force transmission and tendon transfer for patients suffering from spastic paresis: A review and some new observations. Journal of Electromyography and Kinesiology 2007; 17(6):644-656.

7. Hocking DC, Titus PA, Sumagin R, Sarelius IH. Extracellular matrix fibronectin mechanically couples skeletal muscle contraction with local vasodilation. Circulation Research 2008; 102(3):372-379.

8. Heinemeier K, Olesen JL, Haddad F, Langberg H, Kjaer M, Baldwin KM, Schjerling P. Expression of collagen and related growth factors in rat tendon and skeletal muscle in response to specific contraction types. The Journal of Physiology 2007; 582(3):1303-1316.

9. Grinnell F. Fibroblast-collagen-matrix contraction: growth-factor signalling and mechanical loading. Trends in Cell Biology 2000; 10(9):362-365.

\section{Nicoletta Gagliano ${ }^{1}$ \\ Nicola Portinaro 2,3}

1 Department of Biomedical Sciences for Health, Extracellular Matrix Lab, University of Milan, Italy

2 Department of Pediatric Orthopaedic Surgery, IstitutoClinico Humanitas IRCCS, Rozzano, Milan, Italy

3 Department of Medical Biotechnology and Translational Medicine, University of Milan, Italy

\section{Dear Editor,}

We have read with great interest the letter of $\mathrm{Di}$ Lorenzo and coauthors and we thank for the opportunity to write about our research focused on tendons during CP. Many data are available on tendon modifications under different conditions (pathologies, exercises, etc... $)^{1-3}$, but very few data are available on the effects of CP-induced spasticity directly on tendon tissue $e^{4,5}$.Therefore our work has been designed to clarify the structure of tendons in this pathological condition.
We realized such a strong interest Di Lorenzo and coauthors for the muscle-skeletal tissues. This is clearly demonstrated by the cited references in their letter, which are conversely mostly related to studies on muscle tissue modifications in different conditions and, especially, in cerebral palsy (CP).

As Di Lorenzo and coauthors emphasize, three main structures define the mechanics of the musculoskeletal system: skeleton-muscle, connective tissue and bone. In vivo all these structures interact with each others, adapting the body to the mechanics and viceversa.

As a consequence, we completely agree with a multidisciplinary approach to study the effects of CP on the musculoskeletal unit, involving different competencies.

Accordingly, we also agree that a project investigating the alterations of CP in both skeletal muscle and tendons, possibly associated with functional, clinical measures of disability and anatomical architecture of muscles and tendons via ultrasound scanning, would be a very interesting study providing a real comprehensive and deep description of this problem combining biological and clinical approaches.

But this was not the aim of our study, and could be a matter of a new and different study.

In this context we want to point out the statement reported by Di Lorenzo and coauthors that "Heinemeier studies reported that the tendon tissue response, contrary to muscle, is independent of stress levels/contraction type, indicating that this tissue is less sensitive than skeletal muscle to differences in mechanical stimulus".

We disagree with this statement since, despite muscle and tendon can be considered an unique unit, these two tissues are characterized by different embryologic origins, cell types, structure and functions.

Furthermore, several papers showed that mechanical loadings, different in amplitude or in type of strain, elicit different effects on tendon homeostasis ${ }^{6-9}$.

Di Lorenzo and coauthors consider as misleading our statement that " $\mathrm{CP}$ induces relevant modifications of tendons at the molecular level, possibly leading to modifications in order to respond to the increased mechanical loading and increased functional demands induced by spasticity".

Spasticity should be considered as an input acting on tendons, and therefore as a mechanical load very likely eliciting some modifications on tendon connective tissue, according to many previous studies showing that tendons are able to respond to different mechanical loading by modifying their morphological and biomechanical properties to adapt to functional demand.

Our data clearly show that the morphological modifications induced by CP-related spasticity are represented by increased collagen and glycosaminoglycans con- 
tent, hypercellularity, cell rounding, increased vascularity and lipoid degeneration ${ }^{10}$.

Interestingly, we show that this modifications induced on tendons by CP-related spasticity are not simply physiological adaptive responses. In fact, some of the described characteristics in tendons from CP patients are typically occurring in tendinopathy, and this is an unreported finding in the current literature.

Finally we are aware that "thinking outside the box of a basic researcher" is the correct approach, but we are strongly convinced that also our "basic" results could be relevant and, although they are focused on the biology of tendon connective tissue, they contribute to better understand the complexity of CP effects on the musculoskeletal system, especially on tendons.

\section{References}

1. Kjaer M. Role of extracellular matrix in adaptation of tendon and skeletal muscle to mechanical loading. Physiol Rev 2004; 84:649-698.

2. Riley GP. Gene expression and matrix turnover in overused and damaged tendons. Scand J Med Sci Sports 2005; 15:241-251.

3. Docking S, Samiric T, Scase E, Purdam C, Cook J. Rela- tionship between compressive loading and ECM changes in tendons. Muscle, Ligaments and Tendons Journal 2013; 3:711.

4. Gagliano N, Pelillo F, Grizzi F, Picciolini O, Gioia M, Portinaro N. Gene expression profile of extracellular matrix of tendons in cerebral palsy. Dev Med Child Neurol 2007; 49:557558.

5. Gagliano N, Pelillo F, Chiriva-Internati M, et al. Expression profiling of genes involved in collagen turnover in tendons from cerebral palsy patients. Connect Tissue Res 2009; 50:203-208.

6. Lavagnino M, Arnoczky SP. In vitro alterations in cytoskeletal tensional homeostasis control gene expression in tendon cells. J Orthop Res 2005; 23:1211-1218.

7. Lavagnino M, Arnoczky SP, Gardner K. In situ deflection of tendon cell-cilia in response to tensile loading: an in vitro study. J Orthop Res 2011; 29:925-930.

8. Arnoczky SP, Tian T, Lavagnino M, Gardner K. Ex vivo static tensile loading inhibits MMP-1 expression in rat tail tendon cells through a cytoskeletally based mechanotransduction mechanism. J Orthop Res 2004; 22:328-333.

9. Lavagnino M, Arnoczky SP, Tian T, Vaupel Z. Effect of amplitude and frequency of cyclic tensile strain on the inhibition of MMP-1 mRNA expression in tendon cells: an in vitro study. Connect Tissue Res 2003; 44:181-187.

10. Gagliano N, Menon A, Martinelli C, et al. Tendon structure and extracellular matrix components are affected by spasticity in cerebral palsy patients. Muscle, Ligaments and Tendons Journal 2013; 3:42-50. 